\title{
Symptômes neurologiques du bord latéral du pied et de la cheville*
}

\author{
Neurological Symptoms of Outer Side of the Foot or Ankle
}

\author{
F.-C. Wang \\ (C) Lavoisier SAS 2015
}

\section{Rappel anatomique}

Quels sont les nerfs impliqués dans cette région anatomique? Le nerf fibulaire superficiel (NFS) et sa branche cutanée dorsale intermédiaire ou latérale (territoire cutané antérolatéral du pied), le nerf sural (NS) et sa branche terminale, le nerf cutané dorsal latéral du pied (territoire cutané latéral du pied), le nerf calcanéen inférieur (NCI) issu du nerf plantaire latéral (NPL) (innervation motrice du muscle abducteur $\mathrm{du}$ V) et le nerf fibulaire profond (NFP), parfois assisté d'un nerf fibulaire accessoire (innervation motrice du muscle court extenseur des orteil ou pédieux).

Il existe de nombreuses variantes anatomiques, les deux plus fréquentes étant d'une part l'absence de la branche cutanée dorsale intermédiaire du NFS avec prise en charge du territoire cutané par le NS ( $25 \%$ des cas), et d'autre part l'innervation partielle du muscle court extenseur des orteils par un nerf fibulaire accessoire.

\section{Rappel clinique}

\section{Troubles sensitifs}

Quelles sont les plaintes sensitives auxquelles on peut s'attendre?

- Des paresthésies qui sont des sensations cutanées anormales non désagréables spontanées ou provoquées ;

F.-C. Wang $(\bowtie)$

Département de neurophysiologie clinique, CHU Liège, Belgique

e-mail : fc.wang@chu.ulg.ac.be

CHU Sart Tilman B35, B-4000 Liège

* Cet article a fait l'objet d'une présentation lors du congrès SFMCP en 2014.
- des dysesthésies qui sont des sensations cutanées anormales désagréables spontanées ou provoquées ;

- l'allodynie qui est une douleur provoquée par une stimulation normalement non douloureuse ;

- l'hyperalgésie, par opposition à l'hypoalgésie, qui est une réponse anormalement intense à une stimulation douloureuse ;

- l'hyperesthésie, par opposition à l'hypoesthésie, qui est une sensibilité accrue à toute stimulation douloureuse ou non.

Dans la pathologie du pied, il y a quelques particularités. Il faut savoir que ces plaintes sensitives sont augmentées habituellement au lever, à la station debout, à la marche et la nuit. Elles sont diminuées, en revanche, au repos et au déchaussage.

\section{Plaintes motrices}

Il y en a notamment quand les symptômes au niveau du pied résultent d'une pathologie dont le site lésionnel est plus haut situé. On peut avoir un pied tombant dans les radiculopathies L5, les atteintes du tronc commun sciatique ou les atteintes du nerf fibulaire à la tête de la fibula. On peut également avoir une difficulté ou une impossibilité à se mettre sur la pointe des pieds dans les radiculopathies $\mathrm{S} 1$ ou les atteintes du nerf tibial au creux poplité. Lorsque l'atteinte nerveuse est plus distale, en général, il n'y a pas de plainte motrice, bien qu'un testing musculaire analytique bien conduit puisse tout de même montrer un déficit d'extension des orteils (muscle court extenseur des orteils) ou un déficit d'abduction/adduction des orteils (muscles interosseux, lombricaux et abducteur du V). Il y a également, très souvent, des troubles neurovégétatifs.

\section{Tests cliniques pertinents}

Bien entendu le signe de Tinel est fréquemment positif, quelle que soit l'atteinte du tronc nerveux périphérique (NFS au tiers inférieur de la jambe, NFP à la cheville, 
NPE dans le tunnel tarsien, NS à la cheville ou au pied). Dans les atteintes du nerf fibulaire superficiel au tiers inférieur de la jambe, il y a également une manœuvre qui consiste à faire une flexion plantaire associée à l'inversion forcée du pied. Dans les atteintes du NPE ou du nerf tibial dans le tunnel tarsien, il y a la manœuvre de dorsiflexionéversion du pied associée à la dorsiflexion maximale des orteils.

\section{Étiologies}

Le plus souvent, l'atteinte n'est pas au pied ni à la cheville, elle est bien plus haut située. Il s'agit très souvent d'une radiculopathie. Quand les plaintes sont dans le territoire du NFS, c'est une radiculopathie L5. Quand les plaintes sont dans le territoire du NS ou du NPL, il s'agit d'une radiculopathie S1. Moins fréquentes sont les atteintes du tronc commun sciatique. Les atteintes du tronc commun sciatique ont cette particularité de prédominer très nettement dans le territoire du nerf fibulaire par rapport au territoire du nerf tibial. Il peut également s'agir d'une atteinte du nerf fibulaire commun à la tête de la fibula ou d'une atteinte du nerf tibial au creux poplité. Et puis, il faut évidemment penser à toutes les atteintes inflammatoires, non compressives, dont les mononévrites et les multinévrites inflammatoires dans le cadre notamment d'une vascularite (périartérite noueuse). Il ne faut pas oublier non plus les syndromes compartimentaux. Enfin, on en arrive seulement aux neuropathies périphériques tronculaires focalisées soit sur le NFP, le NFS, le NS et le NPL, voire de sa branche, le NCI. Faire un listing complet de toutes les causes d'atteinte de ces nerfs périphériques serait très fastidieux ; nous préférons vous renvoyer aux travaux de Bouysset et al. [1]. Personnellement, je n'en citerai que quelques-unes. Pour le NFP, il faut d'abord songer aux atteintes proximales, donc partielles, du nerf fibulaire commun, par exemple à la fibula ou à la partie supérieure de la jambe. Ce sont parfois des causes iatrogènes à la suite d'une arthroscopie ou d'une ostéotomie. Il y a aussi des causes tumorales (kyste, ostéochondrome) et traumatiques (traction ou compression externe). Dans les atteintes plus distales, on a les problèmes liés à des conflits avec des chaussures trop serrées ou à lanières, souvent associés au port de hauts talons. Il faut également citer les coups répétés sur le dos du pied, notamment dans la pratique de certains sports comme le football. Enfin, il peut exister une compression sous le feuillet supérieur du retinaculum des tendons extenseurs qu'on a appelés improprement « syndrome du canal tarsien antérieur ». Pour le NS, on retrouve des causes traumatiques, iatrogènes (biopsie neuromusculaire) ou non, tumorales et une compression externe par une chaussure ou une chaînette trop serrée. Citons également un syndrome canalaire, très rare, lors de la traversée du fascia profond à mi-jambe ou un petit peu plus distalement. Pour le NFS, on peut aussi envisager les étiologies de façon étagée. D'abord à la tête de la fibula, avec une atteinte partielle du nerf fibulaire commun. Encore une fois, c'est important, dans l'examen d'un patient se plaignant de paresthésies du pied, de toujours effectuer un testing des muscles long et court fibulaires. Au tiers inférieur de la jambe, lorsque le NFS perfore le fascia, il peut y avoir un véritable syndrome canalaire. Il peut également y avoir, à ce niveaulà, des traumatismes locaux (parfois iatrogènes : biopsie neuromusculaire), des étirements nerveux par entorse de la cheville, des tumeurs, une hernie musculaire ou une compression par des chaussures hautes trop serrées. De même, au niveau de la cheville et du pied, on retrouve les coups répétés sur le dos du pied, dans le football notamment, les conflits avec la chaussure, surtout quand il y a un facteur favorisant supplémentaire comme par exemple un pied creux, un tarse bossu ou une bande aponévrotique. Pour le NPL, il peut s'agir d'un syndrome du canal tarsien se limitant au territoire du NPL ou d'une atteinte plus distale : tumeur, traumatisme ou éventuellement un véritable enclavement dans le canal du muscle abducteur. Et enfin, concernant le NCI (ou nerf de l'abducteur du V, ou nerf de Baxter), ce tronc nerveux peut être étiré lors de son horizontalisation à hauteur du carré plantaire ou être comprimé par une hypertrophie du muscle abducteur de l'hallux ou par d'autres structures comme un muscle accessoire, une « épine » calcanéenne ou chez des sportifs (athlètes, joggers) un épaississement de l'aponévrose plantaire.

\section{Rappel de neurophysiologie}

C'est facile d'étudier le NS. Il n'y a pas lieu de penser ou de dire que cet examen est inintéressant ou qu'il n'apporte aucune réponse à des pathologies concernant ce nerf. On réalise une étude dans deux segments, un segment proximal et un segment plus distal correspondant au nerf cutané dorsal latéral du pied qui est la branche terminale du NS. Dans un premier temps, on place une détection des influx nerveux (électrodes de surface) à hauteur de la cheville, et on réalise ensuite une stimulation proximalement à $8 \mathrm{~cm}$ de la détection. C'est donc une stimulation percutanée du NS dans sa partie supérieure, du moins quand on considère le segment cheville-pied. On enregistre ainsi une réponse très facilement de très belle amplitude, et une vitesse de conduction sensitive. Ensuite, on va étudier le segment plus distal. On place la détection $1 \mathrm{~cm}$ distalement par rapport à la base $\mathrm{du} 5^{\mathrm{e}}$ métatarsien et on stimule le NS proximalement à $8 \mathrm{~cm}$ de la détection. On augmente de façon progressive l'intensité de la stimulation nerveuse et on enregistre de nouveau un potentiel qui est un peu plus petit parce qu'on est dans un territoire plus distal. On s'assure qu'on n'a pas de 
meilleure réponse en déplaçant un petit peu dorsalement l'électrode de détection et qu'on a enregistré le potentiel le plus ample possible. Techniquement, c'est assez simple et très reproductible. Voici l'exemple d'une patiente opérée d'un lipome kystique intra-osseux au niveau du calcanéus. Elle présente une hypoesthésie sous-jacente à la cicatrice au niveau du bord latéral du pied. Quand on étudie le NS dans sa partie supérieure, on a des réponses parfaitement symétriques gauche-droite. Du côté de la lésion, quand on étudie le nerf cutané dorsal latéral du pied, il existe une asymétrie très nette de l'amplitude des réponses sensitives évoquées. On arrive donc à très bien documenter cette atteinte du NS dans sa portion distale.

\section{Conclusion}

La pathologie du bord latéral du pied est complexe mais grâce à un examen clinique précis et aux données de l'ENMG, il est possible de la démembrer et de porter un diagnostic précis pouvant déboucher sur une action thérapeutique efficace.

\section{Référence}

1. Bouysset M, Delmi M, Morvan G (2014) Le pied et la cheville. De la clinique aux examens complémentaires. Sauramps, Montpellier 